\title{
Effect of differently worded messages about alcohol on intent to breastfeed among university women in South Korea: a survey
}

Ted Greiner, $\mathrm{PhD}$

Retired, former Professor of Nutrition

Department of Food and Nutrition

Hanyang University

Seoul, South Korea

Email: tedgreiner@yahoo.com

Igor Z. Zubrzycki PhD

Deparment of Animal Molecular Biology

National Research Institute of Animal Production

Balice, Poland

Bo Seul Jegal, MD

Seoul, South Korea

Sang-Sun Lee, $\mathrm{PhD}$

Professor of Nutrition, Emeritus

Department of Food and Nutrition

Hanyang University

Seoul, South Korea 


\section{Abstract}

Background: There is concern that advice not to drink alcohol could discourage the initiation and particularly shorten the duration of breastfeeding. However, little research has explored the impact of variously worded messages about drinking while breastfeeding on women's intentions to drink or to breastfeed. Methods: We haphazardly allocated a convenience sample of nulliparous (>95\%) Korean female university students to be exposed to three differently worded messages on alcohol use during breastfeeding in the body of a questionnaire. Translated to English, they were as follows: Liberal: "Breastfeeding is compatible with moderate but not heavy drinking of alcohol." Moderate: "You can use alcohol while breastfeeding, but only on rare occasions and only 1-2 drinks at a time. Too much alcohol can harm the breastfeeding process. After each drink, you should wait 2 hours before breastfeeding to keep the child from being affected.” Strict: "You should never use alcohol while you are breastfeeding.” Results: Most of the 280 valid responses expressed an intention to breastfeed. Over $90 \%$ of sampled women drank alcohol currently; 91\% of them intended to reduce consumption during breastfeeding. How strictly messages were worded did not affect intention to drink during breastfeeding. Neither strict nor liberal messages reduced intention to drink alcohol while breastfeeding. The strict message clearly told the students what to do, whereas the liberal message left choices up to them. Receiving the liberal message that required the women to decide for themselves reduced their intention to breastfeed $(p<0.001)$. Conclusions: In this sample of largely nulliparous university women, a more strictly worded message regarding alcohol use during breastfeeding did not reduce breastfeeding intentions. Messages that left the decision up to the women did. The risk of harm associated with alcohol consumption during breastfeeding should be weighed against the risk that raising concerns about alcohol consumption might reduce the initiation or duration of breastfeeding. Research is needed to test such messages in different cultures and to follow up after delivery to see if indeed expressed intent correlates with actual practices. Until then, messages regarding breastfeeding and alcohol may still be based only on the unexamined assumptions as to whether cautions about alcohol will affect women's breastfeeding practices.

Key words: alcohol consumption, breastfeeding, health education, message design, exclusive breastfeeding, intent to breastfeed 


\section{Introduction}

Alcohol use during pregnancy is usually strongly advised against because of its proven harmful effects on the fetus (Foltran et al. 2011). Excessive alcohol use by mothers, irrespective of how they feed their infants, is a routine issue for social services in deciding whether alternative arrangements are required for child care (Lewis 1995). The issues regarding moderate use of alcohol during breastfeeding are less clear and messages to mothers are less consistent.

Often the impact of alcohol on the breastfeeding child is said to be minimal. In the past its effects on the breastfeeding process were widely and incorrectly thought be positive. This belief still exists. Thus, health workers sometimes encourage breastfeeding women to drink alcohol (especially beer) (Mennella 2002). One Australian study found that women who attended antenatal classes were more likely to drink alcohol during breastfeeding than those who did not (Giglia et al. 2008). Breastfeeding organizations have provided a range of recommendations on alcohol consumption, sometimes worded largely so as to avoid making breastfeeding sound more complex or difficult to fit into a woman's lifestyle (Wall 2001).

A review in the previous issue of World Nutrition (https://worldnutritionjournal.org/index.php/wn/article/view/623) found that approximately half of breastfeeding women drink alcohol, varying from 83\% to 9\% (Greiner 2019). The same review found that two studies showed no relationship between alcohol consumption and breastfeeding initiation. 15 found no relationship between alcohol consumption and the duration of breastfeeding; three found a negative association and two a positive association. However, while one showed no association and one a positive association, seven found a negative association between the duration of exclusive breastfeeding and concurrent maternal alcohol consumption.

Research on the impact of maternal alcohol consumption during breastfeeding (also reviewed by Greiner (2019) is very limited, with very few findings tested by even a second study. A few studies do raise concerns. One study examined the impact on mothers of taking a single standard drink (resulting in a BAC of 0.03) and found they more often missed infant cues and there was a non-significant increase in dyadic conflict $(\mathrm{p}=0.07)$ (Schuetze, Eiden, and Chan 2002).

It appears that alcohol has mild to moderate disruptive effects on infant sleep (Mennella and Gerrish 1998), fussiness and startles (Schuetze, Eiden, and Chan 2002). One study found potential links with abstract reasoning at 6-7 years (Gibson and Porter 2018) and another with multiple developmental disorders, including a six times greater risk of fetal alcohol spectrum disorders (May et al. 2016). Infants exposed to breastmilk alcohol more readily accept and play with toys soaked in ethanol (Mennella and Beauchamp 1998).

Alcohol inhibits oxytocin release, resulting in less infant breastmilk intake despite increased suckling in the following hours (Mennella and Beauchamp 1993). The resulting fuller feeling in the mother's breasts may account for the widespread belief that alcohol increases breastmilk production (Mennella and Garcia-Gomez 2001). If drinking is only occasional, infants compensate for any reduced intake of breastmilk (Mennella 2001) and also for lost sleep (Mennella and Garcia-Gomez 2001).

The few studies that have been done suggest that knowledge about breastfeeding and alcohol use is low among both health professionals (Mennella 2002) and mothers (Shaker, Scott, and Reid 2004). Regarding attitudes, one review found widely differing results in the proportion of study samples who agreed with the statement "Women who occasionally drink alcohol should not breastfeed,” varying from 23\% to 76\% (Greiner 2019). 
In one study, women who were advised not to drink during breastfeeding did so less than those who were encouraged to drink or not given any advice (Mennella 1997). However, it is challenging to decide how liberal or restrictive advice about breastfeeding and moderate use of alcohol should be, since harm could in theory result from messages that either give the impression that breastmilk alcohol is less harmful than recent research suggests it may be (and thus provide no incentive to reduce consumption and/or infant exposure) or messages that alarm women who drink occasionally to the point where they unnecessarily avoid or shorten the period of breastfeeding.

Thus, we decided to test the potential impact of messages that were restrictively worded (strict), complex but scientifically accurate (moderate), or more laissez-faire (liberal) about breastfeeding and alcohol. We compared how such messages might affect intentions to drink alcohol during breastfeeding to breastfeed among largely nulliparous university women in Seoul, South Korea.

\section{Subjects and Methods}

\section{Sample}

A convenience sample of female graduate and undergraduate students in nutrition classes at Hanyang University, Seoul, South Korea were asked if they were willing to volunteer to fill in questionnaires related to breastfeeding perceptions during April, 2010. Deciding that a larger sample size was needed, the questionnaire was administered again in September, 2010 to classes in other departments.

\section{Methods}

Respondents were asked not to place their names on the questionnaires (which were in Korean for all but a few students who requested them in English), and thus were aware that their responses were anonymous. They were not told that three different types of questionnaires were being haphazardly distributed among them. After they had responded to nine identical background questions, the questionnaire presented them with the following text (translated from the Korean):

Imagine you were pregnant. You have sought advice from the single person you trust most. This might be a doctor or other health worker, or someone you believe is wise about how to raise a healthy baby. The following two questions ask about how you imagine you would feel if that person said the following to you:

Then one the following three statements was presented (only one of these statements was on each questionnaire):

1. "Breastfeeding is compatible with moderate but not heavy drinking of alcohol."

2. "You can use alcohol while breastfeeding, but only on rare occasions and only 1-2 drinks at a time. Too much alcohol can harm the breastfeeding process. After each drink, you should wait 2 hours before breastfeeding to keep the child from being affected."

3. "You should never use alcohol while you are breastfeeding" 
An example of the questionnaire (liberal statement) is provided in the Annex.

We characterized the wording of these three statements respectively as liberal, moderate (but also more scientifically worded and complex than the others) and strict. The students were asked not to talk with each other while filling out the form. After the questionnaires were filled in, which took only a few minutes, they were all collected and then we informed the students that each person had received one of three statements, and explained the issues, including the fact that the "moderate" statement was the most accurate of the three.

\section{Data analysis}

We used Chi Square within the Statistical Package for the Social Sciences to test differences between the three groups, considering $\mathrm{p}<0.05$ to be significant. A sample size of 130 was needed to achieve $90 \%$ power (Lenth 2009), but we found this gave too many cells of sample size $<5$ and thus decided to double the sample size.

The Hanyang University Institutional Review Board approved this study. Student participation was voluntary.

\section{Results}

\section{Participant characteristics}

There were 280 completed questionnaires used in this study. There were no refusals, but 9 other questionnaires were not used because the respondent marked that she was certain she would never have a child (in this case, respondents were instructed not to complete the rest of the questionnaire). The mean age of the respondents was 23.3 years ( $\mathrm{SD} \pm 3.2$, minimum 18, maximum 34). Five of these women had one child, one had two children and the rest had no children. Eight of the respondents knew they were pregnant, one of whom already had one child. The respondents had 15 years of education on average and rated their families as middle class on average. Table 1 compares some background data among the three groups. There were no statistically significant differences (using Anova and posthoc testing with Bonferroni), suggesting that the haphazard distribution of the questionnaires was successful in avoiding selection bias.

Table 1. Background characteristics by type of questionnaire filled in; mean (95\% confidence intervals)

\begin{tabular}{|l|l|l|l|}
\hline & Strict questionnaire & $\begin{array}{l}\text { Moderate } \\
\text { questionnaire }\end{array}$ & Liberal questionnaire \\
\hline Age (years) & $23.3(22.7-23.9)$ & $23.4(22.7-24.1)$ & $23.4(22.7-24.0)$ \\
\hline $\begin{array}{l}\text { Years of education } \\
\text { completed }^{\text {Income level }}\end{array}$ & $14.9(14.6-15.2)$ & $15.0(14.6-15.4)$ & $15.1(14.7-15.5)$ \\
\hline Number of children $^{\mathrm{a}}$ & $2.85(2.68-3.20)$ & $2.98(2.82-3.14)$ & $2.93(2.77-3.09)$ \\
\hline
\end{tabular}

${ }^{\mathrm{a}}$ Coding: low $=1$, low-middle $=2$, middle $=3$, high-middle $=4$, high $=5$. 
As shown in Table 2, the total sample was rather evenly divided among women who wanted one child or less in the future, those who wanted to have at least one child, and those who wanted to have more than one child.

Table 2. Do you want children in the future?

\begin{tabular}{|l|l|l|}
\hline Responses $(\mathrm{N}=279)$ & Number & Percent \\
\hline Never $^{\mathrm{a}}$ & 16 & 5.7 \\
\hline I doubt it & 36 & 12.9 \\
\hline I don't know & 33 & 11.8 \\
\hline Only one & 16 & 5.7 \\
\hline One or more & 92 & 33.0 \\
\hline More than one & 86 & 30.8 \\
\hline
\end{tabular}

a These women were included because they did not mark "I am certain I will never have a child" and thus completed the questionnaire

\section{Breastfeeding and alcohol consumption intentions}

Before coming to the message about drinking and breastfeeding, women were asked about how they might feel about breastfeeding if they had a child. Table 3 (from now on, also leaving out the 16 who said they never wanted to have a child and 4 others who did not complete the entire questionnaire) reports on their responses by message category. Similar answers were given for each of the three groups at baseline, with very few intending not to breastfeed. There are again no differences by type of message. Even if we combined the three lowest categories in the table to eliminate small cells, the Chi Square value for the null hypothesis of equality between the three groups was only $6.7(p=0.6)$.

Table 3. How would you feel about breastfeeding if you had a child? ${ }^{\mathrm{a}}$

\begin{tabular}{|l|l|l|l|l|}
\hline \multirow{2}{*}{ Amount of breastfeeding } & \multicolumn{2}{l|}{ Number (per cent) by type of message } & Total Number \\
\cline { 2 - 5 } & Strict & Moderate & Liberal & (per cent) \\
\hline As long as possible & $54(60)$ & $58(65)$ & $52(65)$ & $164(64)$ \\
\hline For a short period & $21(23)$ & $18(20)$ & $19(24)$ & $58(22)$ \\
\hline Unsure & $14(16)$ & $11(12)$ & $9(11)$ & $34(13)$ \\
\hline Probably not & 0 & $2(2.3)$ & 0 & $2(0.8)$ \\
\hline Definitely not & $1(1.1)$ & 0 & 0 & $1(0.4)$ \\
\hline Total & 90 & 89 & 80 & 259 \\
\hline
\end{tabular}

a Those who marked that they wanted no children were not asked to answer this question

${ }^{\mathrm{b}} \mathrm{Chi}$ Square for the null hypothesis of equality between the three groups $=6.7(\mathrm{p}=0.6)$.

Table 4 suggests that the three groups also had similar self-described current alcohol consumption patterns (Chi Square 8.0, $\mathrm{p}=0.4$ ). Overall, 54\% were drinking either never or less than once a month and another $28 \%$ were drinking more than once a month but less than once a week. 
Table 4. How often do you drink alcohol?

\begin{tabular}{|c|c|c|c|c|}
\hline \multirow{2}{*}{$\begin{array}{l}\text { Frequency of } \\
\text { drinking }\end{array}$} & \multicolumn{3}{|c|}{ Number (per cent) by type of message } & \multirow{2}{*}{$\begin{array}{l}\text { Total Number } \\
\text { (per cent) } \\
\text { Total }\end{array}$} \\
\hline & Strict & Moderate & Liberal & \\
\hline Never & $9(9.8)$ & $8(8.8)$ & $7(8.0)$ & $24(8.9)$ \\
\hline$<1$ drink a month & $38(42)$ & $40(44)$ & $44(51)$ & $122(45)$ \\
\hline$<1$ drink a week & $29(32)$ & $20(22)$ & $25(29)$ & $74(28)$ \\
\hline 1-4 drinks per week & $6(6.6)$ & $10(11)$ & $7(8.0)$ & $23(8.6)$ \\
\hline 5-7 drinks per week & $8(8.7)$ & $12(13.3)$ & $3(3.4)$ & $23(8.6)$ \\
\hline$>1$ per day & $1(1.1)$ & $1(1.1)$ & $1(1.1)$ & $3(1.1)$ \\
\hline Total & 91 & 91 & 87 & 269 \\
\hline
\end{tabular}

${ }^{\mathrm{a}}$ Chi Square for the difference between the three groups $=8.0(\mathrm{p}=0.4)$

All the previous questions were asked before the statement about breastfeeding and alcohol consumption (liberal, moderate, or strict) in the questionnaire. After reading the statement, women were asked to estimate how much they might drink during breastfeeding. Table 5 shows that nearly all the respondents intended either not to drink or to drink very rarely while breastfeeding. The slight apparent differences in the expected direction (less intended drinking with more strict messages) were not statistically significant (Collapsing into 3 categories, Chi Square=6, $\mathrm{p}=0.19$; test for linearity, $\mathrm{p}=0.07$ ).

Table 5. How often would you expect to drink alcohol if you were breastfeeding? ${ }^{\mathrm{a}}$

\begin{tabular}{|l|l|l|l|l|}
\hline \multirow{2}{*}{$\begin{array}{l}\text { Frequency of } \\
\text { drinking }\end{array}$} & Number (per cent) by type of message & \multicolumn{1}{|c|}{$\begin{array}{c}\text { Total } \\
\text { Number (per } \\
\text { cent) Total }\end{array}$} \\
\cline { 2 - 5 } & Strict & Moderate & Liberal & $199(80)$ \\
\hline Never & $73(85)$ & $67(79)$ & $59(76)$ & $44(18)$ \\
\hline$<1$ drink a month & $11(13)$ & $19(22)$ & $14(18)$ & $4(1.6)$ \\
\hline$<1$ drink a week & $1(1.2)$ & $1(1.2)$ & $2(2.6)$ & $2(0.8)$ \\
\hline $1-4$ drinks per week & 0 & 0 & $2(2.6)$ & 0 \\
\hline 5-7 drinks per week & 0 & 0 & 0 & 0 \\
\hline $\begin{array}{l}>1 \text { per day on } \\
\text { average }\end{array}$ & 0 & 0 & 0 & 249 \\
\hline Total & 86 & 85 & 78 & \\
\hline
\end{tabular}

${ }^{a}$ Those who marked that they wanted no children were not asked to answer this question

${ }^{\mathrm{b}}$ Chi Square for difference between the three groups $=6, \mathrm{p}=0.19$; test for linearity, $\mathrm{p}=0.07$

Table 6 reports on women's responses regarding how the message they received on drinking and breastfeeding might change their intentions to breastfeed if they fully trusted that this was wise advice. The more liberal the message, the more respondents indicated that it might result in their breastfeeding less ("It might make me hesitate to breastfeed, or at least would likely result in my breastfeeding less") or not at all (Chi Square=32.7, $\mathrm{p}<0.001$ ). Leaving out from the analysis those who currently never drink reduces the sample size to 228, but the pattern remains the same; the Chi Square reduces to 19.4 but is still significant at $\mathrm{p}<0.001$. 
Table 6 . How would the message about alcohol affect the likelihood of your breastfeeding? ${ }^{\mathrm{a}}$

\begin{tabular}{|l|l|l|l|l|}
\hline \multirow{2}{*}{ Likely change } & \multicolumn{3}{|l|}{ Number (per cent) by type of message } & \multicolumn{2}{l|}{$\begin{array}{l}\text { Total Number } \\
\text { (per cent) Total }\end{array}$} \\
\cline { 2 - 5 } & Strict & Moderate & Liberal & $158(65)$ \\
\hline None & $64(78)$ & $61(74)$ & $31(41)$ & $90(36)$ \\
\hline Would breastfeed less & $16(20)$ & $21(25)$ & $35(46)$ & $13(5.4)$ \\
\hline $\begin{array}{l}\text { Would probably not } \\
\text { breastfeed }\end{array}$ & $2(2.4)$ & $1(1.2)$ & $10(13)$ & 241 \\
\hline Total & 82 & 83 & 76 & \\
\hline
\end{tabular}

${ }^{a}$ Those who marked that they wanted no children were not asked to answer this question

${ }^{\mathrm{b}}$ Chi Square for the difference between the three groups $=32.7, \mathrm{p}<0.001$

\section{Discussion}

We found that nearly all the young women in our sample of largely nulliparous Korean university students who wanted to have children expected they would breastfeed. Over 90\% currently consumed alcohol but, among them, 79\% planned never to drink while breastfeeding and another $12 \%$ to reduce it. More strictly worded messages were associated with the expectation to drink less during breastfeeding but this difference was non-significant.

Here is a translation of the advice on drinking alcohol while breastfeeding from the Food and Drug Administration of Korea (KFDA): "It is okay to drink alcohol from time to time but drinking every day or getting drunk makes a breastfed baby sleepy or disturbs the growth of the motor nerves. It also reduces the secretion of the breastmilk. If a mother still wants to drink badly, she must limit drinking to less than twice a week. After drinking she has to wait for at least two hours before breastfeeding."

The moderately worded message was more complex and required a specific set of activities. This may have resulted in women deciding they would breastfeed less, compared to those who received a very simple, strict message. The finding that women exposed to the liberal message ("Breastfeeding is compatible with moderate but not heavy drinking of alcohol.") were more likely to respond that this would lead them to breastfeed less was unexpected. We hypothesize that the language used in the KFDA recommendation may be the reason for this. The other two messages, like the KFDA message, give very specific guidance on exactly what the mother should do. Being told exactly what to do is expected by a young woman in the Korean culture. The liberal message unintentionally broke from that convention and left it up to the mother to define what moderate vs. heavy drinking would be. This may have made any that did want to drink while breastfeeding feel so insecure that they decided it would be safer to reduce how much they breastfed or not to breast feed at all. Thus, in a sense we made a similar mistake to researchers who use the term "responsible drinking” without adequately defining it (Barry and Goodson 2010).

This study should be viewed as preliminary. Any social research conducted on a convenience sample, as this one was, cannot be safely generalized to any broader population. We know of no reasons why this sample would not have been "typical" of female Korean undergraduate students. For example, the frequency of drinking we observed was similar or perhaps slightly less than that reported from a smaller sample of university women from the Gangwon area (Chung and Lee 2012) and about the same as that reported for an equally large sample of female students from Shingyeong University, Hwaseong (Ryu and Baek 2012). While it was possible that some students, had heard about the questionnaire from others who had taken 
it previously, we believe this is unlikely and would not have been common enough to affect our results. In particular, those administered a half year later were administered to students in other departments. However, we cannot rule out that our sample was somehow biased and thus would caution against generalizing from it to all Korean university women.

Based perhaps on myths that alcohol or alcohol-containing beer enhances lactation or on early (but somewhat incorrect) research findings of little or no impact of alcohol on breastfeeding or on the infant, many in the "breastfeeding community" believe that quite liberal messages should be given to women on the question of whether they can drink while breastfeeding. There is an understandable concern that more restrictive or heavy-handed messages will make breastfeeding appear to be burdensome, reducing the proportion of women who initiate breastfeeding, how intensively they breastfeed, or the duration of breastfeeding (each of which could have a negative public health impact). However, organizations that keep up to date are publishing slightly more restrictive advice than in the past. See for example La Leche League's statement (La Leche League International 2018).

In contrast, many public health officials, concerned more about alcohol consumption than breastfeeding, may tend to advise categorically against alcohol consumption during breastfeeding (U.S. Department of Health and Human Services and U.S. Department of Agriculture 2005). Probably messages should be placed somewhere between these extremes, but virtually no research has been done testing what kinds of wording of messages might provide correct information about potential harm, how to drink in ways that would reduce it, and yet not unduly discourage breastfeeding.

The present study suggests a preliminary method for testing how messages with stricter or more liberal wording change intentions to drink during breastfeeding and intentions to breastfeed. The English version of the questionnaire used (with the liberal message; obviously the others used the strict or moderate messages quoted above) is presented as an annex to this paper and the Korean version will be supplied on request to the first author. To increase the chances of there being any impact, we used somewhat exaggerated wording in the strict and liberal messages and presented the more correct and scientific information to the respondents after all had completed the questionnaire. We do not know if any of these students already were familiar with either scientific or unscientific information on this topic, but discussions after they had filled in the questionnaire suggest few were.

It should be kept in mind that there is often a difference between behavioral intentions and actions; often intentions explain only about half of the variance in actual behavior. Similarly, a single simplistic message seen on a single occasion should not be expected to have a large impact on intention, let alone behavior.

In conclusion, we found that how strictly messages on alcohol use during breastfeeding were worded to largely nulliparous Korean female university students had little impact on their already restrictive intentions to drink during breastfeeding. Far from inhibiting breastfeeding, for some reason the more strictly worded the message was (recommending little or no drinking during breastfeeding), the less likely it was to lead these women to intend to breastfeed less.

For ethical reasons, testing the impact of such messages in real life trials involving the measurement of actual—or more accurately, stated--behavior would require the use of correct messages, thus limiting the differences among them. Testing the impact of very small differences in different accurately worded messages would certainly require large sample sizes. In any case, until further study of the impact of messages on alcohol use during breastfeeding is done, such messages will continue to be based solely on assumptions as to their possible impact. Our 
research suggests that assumptions about the impact of messages about breastfeeding and alcohol consumption may often be incorrect.

\section{Acknowledgements}

The authors are grateful to the women who participated in this study and our fellow teachers for giving us access to them. No funding was received to do this research.

\section{Competing interests}

The authors declare that they have no competing interests.

\section{Authors' contributions}

TG designed the study, did the data analysis, and did most of the writing. IZZ assisted with statistical planning and writing. BSJ coded the data and assisted with the literature review. SSL assisted in planning the study and interpretation of the results. All authors have seen and approved the final version of this paper.

\section{References}

Barry, Adam E., and Patricia Goodson. 2010. "Use (and Misuse) of the Responsible Drinking Message in Public Health and Alcohol Advertising: A Review." Health Education \& Behavior 37 (2):288303. doi: 10.1177/1090198109342393.

Chung, H. K., and H. Y. Lee. 2012. "Drinking behaviors by stress level in Korean university students." Nutr Res Pract 6 (2):146-54.

Foltran, FF, DF Gregori, LF Franchin, EF Verduci, and M. Giovannini. 2011. "Effect of alcohol consumption in prenatal life, childhood, and adolescence on child development." Nutr Rev 69 (11):642-59 doi: LID - 10.1111/j.1753-4887.2011.00417.x

Gibson, Louisa, and Melanie Porter. 2018. "Drinking or Smoking While Breastfeeding and Later Cognition in Children." Pediatrics e20174266. doi: 10.1542/peds.2017-4266.

Giglia, R. C., C. W. Binns, H. S. Alfonso, J. A. Scott, and W. H. Oddy. 2008. "The effect of alcohol intake on breastfeeding duration in Australian women." Acta Paediatrica 97 (5):624-629. doi: 10.1111/j.1651-2227.2008.00760.x.

Greiner, Ted. 2019. "Alcohol and Breastfeeding, a review of the issues." World Nutrition 10 (1):63-88.

La Leche League International. 2018. "Drinking alcohol and breastfeeding." accessed March 19, 2018. https://www.llli.org/breastfeeding-info/alcohol/.

Java Applets for Power and Sample Size [Computer software]. .

Lewis, KD. 1995. Infants and Children with Prenatal Alcohol and Drug Exposure: A Guide to Identification and Intervention. North Branch, MN: Sunrise River Press.

May, P. A., J. M. Hasken, J. Blankenship, A. S. Marais, B. Joubert, M. Cloete, M. M. de Vries, R. Barnard, I. Botha, S. Roux, C. Doms, J. P. Gossage, W. O. Kalberg, D. Buckley, L. K. Robinson, C. M. Adnams, M. A. Manning, C. D. Parry, H. E. Hoyme, B. Tabachnick, and S. Seedat. 2016. "Breastfeeding and maternal alcohol use: Prevalence and effects on child outcomes and fetal alcohol spectrum disorders." Reprod Toxicol 63:13-21. doi: 10.1016/j.reprotox.2016.05.002.

Mennella, J A. 2001. "Regulation of milk intake after exposure to alcohol in mothers' milk." Alcohol Clin Exp Res 25 (4):590-593.

Mennella, J. A. 1997. "Infants' suckling responses to the flavor of alcohol in mothers' milk." Alcohol Clin Exp Res. 21 (4):581-5.

Mennella, J. A., and G. K. Beauchamp. 1993. "Beer, breast feeding, and folklore." Dev Psychobiol 26 (8):459-466. 
Mennella, J. A., and G. K. Beauchamp. 1998. "Infants' exploration of scented toys: Effects of prior experiences." Chemical Senses 23:11-17.

Mennella, J. A., and P. L. Garcia-Gomez. 2001. "Sleep disturbances after acute exposure to alcohol in mothers' milk." Alcohol 25 (3):153-158.

Mennella, J. A., and C. J. Gerrish. 1998. "Effects of exposure to alcohol in mother's milk on infant sleep." Pediatrics 101 (5):E2.

Mennella, J.A. 2002. "Alcohol and lactation: the folklore versus the science." In Current issues in clinical lactation, edited by K.G. Auerbach, 3-10. Boston: Jones and Bartlett.

Ryu, H-S., and M-J. Baek. 2012. "Factors influencing the drinking behavior in female university students." J Korean Acad Community Health Nurs 23 (3):307-315.

Schuetze, Pamela, Rina Das Eiden, and Arthur W. K. Chan. 2002. "The effects of alcohol in breast milk on infant behavioral state and mother-infant feeding interactions." Infancy 3 (3):349-363. doi: 10.1207/s15327078in0303_4.

Shaker, I., J. A. Scott, and M. Reid. 2004. "Infant feeding attitudes of expectant parents: breastfeeding and formula feeding." Journal of Advanced Nursing 45 (3):260-268.

U.S. Department of Health and Human Services, and U.S. Department of Agriculture. 2005. Dietary Guidelines for Americans. 6th ed. Washington DC: U.S. Government Printing Office.

Wall, G. 2001. "Moral constructions of motherhood in breastfeeding discourse." Gender \& Society 15 (4):592-610. doi: 10.1177/089124301015004006. 


\section{Annex 1. Questionnaire_Liberal}

This questionnaire is to study perceptions of breastfeeding among young women in Korea. This anonymous questionnaire could be very useful material to improve our understanding of these issues. We promise that your questionnaire will be used only for research purposes but filling it in is completely voluntary. Thank you for your kind participation.

1. Your birth date

2. How much education have you had?

3. How would you describe your income level? [Answer about your family's income level if you are still living with your parents.]

a. Low income

b. Low-middle income

c. Middle income

d. High-middle income

e. High income

4. Marital status

a. Never been married; not currently engaged to be married

b. Never been married; currently engaged to be married

c. Divorced

d. Husband died

e. Currently married

5. How many children do you have?

6. Are you currently pregnant?

a. No

b. Yes [As you answer this questionnaire, imagine you were pregnant again with another child in the future.]

7. How do you feel about having children in the future?

a. I am certain I will never have any [Thank you for participating in this survey. Please give your questionnaire to the proctor now.]

b. I doubt I will ever have children

c. I am uncertain whether I want to have children

d. I am willing to have one but only one child

e. I would like to have one or more children

f. I would like to have more than one child

8. How do you feel about breastfeeding if you have a child? (or another one if you already have one)

a. I will definitely breastfeed for as long as the child wants

b. I will definitely breastfeed, but only for a short time

c. I am not sure if I will try to breastfeed 
d. I will probably not breastfeed

e. I am certain that I will never try to breastfeed [Thank you for participating in this survey. Please give your questionnaire to the proctor now.]

9. Please choose whichever of the following statements is closest to the way you take alcoholic drinks (a drink is one beer, one small glass of wine, or one shot glass of soju or other strong drink):

a. I never drink alcohol and intend never to start doing so [Skip to question 12]

b. I rarely drink alcohol (<one drink a month)

c. I drink alcohol occasionally (<one drink a week)

d. I take 1-4 drinks in the average week

e. I take 4-8 drinks in the average week

f. I take $1-2$ drinks every day on average

g. I take $>2$ drinks every day

Imagine you were pregnant. You have sought advice from the single person you trust most. This might be a doctor or other health worker, or someone you believe is wise about how to raise a healthy baby. The following two questions ask about how you imagine you would feel if that person said the following to you:

"Breastfeeding is compatible with moderate but not heavy drinking of alcohol."

10. If you fully trusted that this was wise advice:

a. It would have no influence on whether or how long I breast fed my baby

b. It might make me hesitate to breast feed, or at least would likely result in my breastfeeding less

c. After hearing this, I probably would decide not to breastfeed

11. This question asks about how you would drink during breastfeeding if you fully trusted that this was wise advice:

a. I would probably never drink alcohol during breastfeeding

b. I would probably rarely drink alcohol (<one drink a month)

c. I would probably drink alcohol occasionally (<one drink a week)

d. I would probably take 1-4 drinks on the average week

e. I would probably take 4-8 drinks on the average week

f. I would probably take 1-2 drinks every day on average

g. I would probably take $>2$ drinks every day 